\title{
Influence of Weed Management by Allelopathy on Growth, Yield and Nutrient Status of Soil in Pigeonpea (Cajanus cajan L.)
}

\author{
Shridhara $^{1}$, B. Nagoli ${ }^{\text {* }}$, B. M. Dodamani ${ }^{2}$, M. Y. Ajaya Kumar ${ }^{2}$, \\ Pandit S. Rathod, Mahadeva Swamy ${ }^{4}$ and K. Basavaraj ${ }^{5}$ \\ ${ }^{1}$ College of Agriculture, Raichur, India \\ ${ }^{2}$ College of Agriculture, Kalaburagi, India \\ ${ }^{3}$ Main Agriculture Research Station, Raichur, India \\ ${ }^{4}$ Zonal Agriculture Research Station, Kalaburagi, India \\ ${ }^{5}$ University of Agriculture sciences, Raichur, India \\ *Corresponding author
}

\section{A B S T R A C T}

\section{Keywords}

Allelopathy, Available nutrients, 100-seed weight, Grain yield

Article Info

Accepted:

12 December 2020 Available Online: 10 January 2021
A pot experiment was carried out at Zonal Agriculture Research Station, Kalaburagi (Karnataka), during kharif 2018 and 2019 to study the influence of weed management by allelopathy on nutrient status of soil in pigeonpea (Cajanus cajan L.). The soil was medium deep black with soil $\mathrm{pH}(8.13), \mathrm{EC}\left(0.25 \mathrm{dS} \mathrm{m}^{-1}\right)$, available nitrogen $\left(242 \mathrm{~kg} \mathrm{ha}^{-1}\right), \mathrm{P}_{2} \mathrm{O}_{5}(34 \mathrm{~kg}$ $\mathrm{ha}^{-1}$ ) and $\mathrm{K}_{2} \mathrm{O}\left(347 \mathrm{~kg} \mathrm{ha}^{-1}\right)$. The experiment was laid out in completely randomized block design which comprised of 18 treatments with three replications, consisting four plant extracts, i.e., Sorghum, Parthenium, Cassia and Eucalyptus and imazythapyre herbicide. All the practices followed were according to package of practice of UAS, Raichur. The data of each crop season were statistically analyzed. Weed management practices significantly influenced the available nutrients in soil (N, P and K) among all the treatments at harvest of pigeonpea. Imazythapyre @ $56.25 \mathrm{~g}$ a.i./ha + sorghum plant extract at 20-25 DAS, imazythapyre @ $56.25 \mathrm{~g}$ a.i./ha + cassia plant extract at 20-25 DAS, imazythapyre @ 56.25 g a.i./ha + parthenium plant extract at 20-25 DAS, imazythapyre@ 37.5 g a.i./ha + sorghum plant extract at 20-25 DAS, imazythapyre @ $56.25 \mathrm{~g}$ a.i./ha + eucalyptus leaf extract at 20-25 DAS, imazythapyre @ $37.5 \mathrm{~g}$ a.i./ha + cassia plant extract at 20-25 DAS, imazythapyre @ 37.5 g a.i./ha + parthenium plant extract at 20-25 DAS and imazythapyre @ 37.5 g a.i./ha + eucalyptus leaf extract at 20-25 DAS recorded significantly higher number of primary and secondary branches, seed yield per plant, 100 seed weight and grain yield and lower available nitrogen, phosphorus and potassium compared to rest of the treatments. Plant extracts when used with herbicide help to reduce usage of herbicide by $50 \%$. Soil fertility and productivity can be maintained for longer period through which crop production can become more productive, sustainable and economical both directly and indirectly.

\section{Introduction}

Pulses are an important component of agricultural food crops and the cheapest source of dietary protein. The high content of protein in pulses makes the diet more nutritive for vegetarian when consumed with other cooked food items. Pulses are rich source of 
protein, dietary fiber, complex carbohydrates and $\mathrm{K}, \mathrm{Se}, \mathrm{Fe}$ and $\mathrm{Zn}$-power house of nutrients. Pulses are also known for increasing productivity of soil through fixation of nitrogen from atmosphere. Pulses are well suited in rainfed condition and require less farm resources; hence farmers prefer to grow them from economic point of view throughout the country that helps to eliminate hunger, food security and malnutrition. India plays a key role in global pulse production and contributes about 25 per cent to the total pulse basket with total production of 3.38 million tonnes from 4.55 million ha area (Anon., 2019). On account of their value as nutritious food, feed and forage, pulses remained an integral component of subsistence cropping system since time immemorial. Weed is one of the important limiting factor in pigeonpea production through competition for environmental resource and can reduce yield by 30-45 \% (Talnikar et al., 2008). Today, the most of pigeonpea producers use chemical herbicides for weed control in pigeonpea. Application of high dose of chemical herbicide in agricultural production causes many adverse effects that most of them relating to incorrect use of herbicide. One of the adverse effects of herbicides application is evolution of resistant weed biotypes.

The major challenge for India is that of producing adequate food for the ever growing human population. Traditional method of pigeonpea cultivation is no longer a viable system of crop production because of increasing human population on limited arable land which has reduced the duration of fallow, low soil fertility, declining crop yield and high weed pressure are some of the problems that have been associated with reduced fallow duration and intensification of cropping systems. Pigeonpea being long duration crop, cultivated with wider spacing due to which weed management in these rows plays significant role on yield of the crop. These weeds can cause $20-90 \%$ yield loss in pulse crops (Pooniya et al., 2015). Management of weeds by using herbicide is the commonly followed method. Recent advances in management of weeds through plant water extracts i.e., allelopathy is gaining importance due to better control of weeds, cost effective and biodegradability.

\section{Materials and Methods}

A pot experiment was carried out at Zonal Agriculture Research Station, Kalaburagi (Karnataka), during kharif 2018 and 2019; the research was conducted to study the influence of weed management by allelopathy on growth, yield and nutrient status in pigeonpea (Cajanus cajan L.). The soil was medium deep black with soil $\mathrm{pH}$ (8.13), EC (0.25 dS $\left.\mathrm{m}^{-1}\right)$, available nitrogen $\left(242 \mathrm{~kg} \mathrm{ha}^{-1}\right), \mathrm{P}_{2} \mathrm{O}_{5}$ (34 $\mathrm{kg} \mathrm{ha}^{-1}$ ) and $\mathrm{K}_{2} \mathrm{O}$ (347 $\mathrm{kg} \mathrm{ha}^{-1}$ ). The experiment was laid out in completely randomised block design which comprised of 18 treatments in three replications, consisting four plant extracts, i.e., Sorghum, Parthenium, Cassia and Eucalyptus and imazythapyre herbicide. All the practices followed were according to package of practice of UAS, Raichur. Treatments as follows; $\mathrm{T}_{1}$ : Imazythapyre@18.75 g a.i./ha + Sorghum plant extract at 20-25 DAS, $\mathrm{T}_{2}$ : Imazythapyre @ $37.5 \mathrm{~g}$ a.i./ha + Sorghum plant extract at 20-25 DAS, T : Imazythapyre @ 56.25 g a.i./ha + Sorghum plant extract at 20-25 DAS, $\mathrm{T}_{4}$ : Only Sorghum plant extract at 20-25 DAS, T5: Imazythapyre @ 18.75 g a.i./ha + Parthenium plant extract at 20-25 DAS, T6: Imazythapyre @ $37.5 \mathrm{~g}$ a.i./ha + Parthenium plant extract at 20-25 DAS, T 7 : Imazythapyre @ $56.25 \mathrm{~g}$ a.i./ha + Parthenium plant extract at 20-25 DAS, T 8 : Only Parthenium plant extract at 20-25 DAS, T9: Imazythapyre @ $18.75 \mathrm{~g}$ a.i./ha + Eucalyptus leaf extract at 2025 DAS, T 10 : Imazythapyre @ $37.5 \mathrm{~g}$ a.i./ha + Eucalyptus leaf extract at 20-25 DAS, T ${ }_{11}$ : 
Imazythapyre @ 56.25 g a.i./ha + Eucalyptus leaf extract at 20-25 DAS, $\mathrm{T}_{12}$ : Only Eucalyptus leaf extract at 20-25 DAS, $\mathrm{T}_{13}$ : Imazythapyre @ $18.75 \mathrm{~g}$ a.i./ha + Cassia plant extract at 20-25 DAS, T 14 : Imazythapyre @ $37.5 \mathrm{~g}$ a.i./ha + Cassia plant extract at 20-25 DAS, $\mathrm{T}_{15}$ : Imazythapyre @ $56.25 \mathrm{~g}$ a.i./ha + Cassia plant extract at 20-25 DAS, $\mathrm{T}_{16}$ : Only Cassia plant extract at 20-25 DAS, $\mathrm{T}_{17}$ : Weed free and $\mathrm{T}_{18}$ : Unweeded check were used in the experiment..

Recommended dose of N, P and K (25:50:00 $\mathrm{kg} \mathrm{N}, \mathrm{P}_{2} \mathrm{O}_{5} \mathrm{~K}_{2} \mathrm{O} \mathrm{ha}^{-1}$ ) were applied to the soil in the form of urea, di-ammonium phosphate and muriate of potash at the time of sowing and subsequent applications were done by following package of practice. At each spot two seeds were dibbled up to 4 to $5 \mathrm{~cm}$ deep in the seed lines. The pigeonpea variety, TS$3 \mathrm{R}$ was used during both the years and was sown at spacing of $90 \times 30 \mathrm{~cm}$. All agronomical package of practices were followed to raise the crop. Available nitrogen was determined by alkaline potassium permanganate method as described by (Subbaiah and Asija, 1956). Available phosphorus was determined by Olsen's method (Jackson, 1973) using Systronics UV visible Spectrophotometer (Model: 117). Available potassium was extracted with neutral ammonium acetate $(\mathrm{pH} 7.0)$ and the content of potassium in the solution was estimated by Systronics Flame Photometer (Model: 128) (Jackson, 1973).

The data of each crop season were statistically analyzed. Fisher's method of analysis of variance was applied for analysis and interpretation of the data as given by Panse and Sukhatme, 1967. The level of significance used in ' $\mathrm{F}$ ' test was at $\mathrm{p}=0.05$. Critical difference values were calculated whenever ' $F$ ' was significant. In other cases, values of standard error of means have been provided.

\section{Results and Discussion}

\section{Number of primary branches}

The weed free treatment remarked significantly more number of primary branches (14.19) at harvest (Table 1) and was on par with imazythapyre @ $56.25 \mathrm{~g}$ a.i./ha + sorghum plant extract at 20-25 DAS (14.10), imazythapyre@56.25 g a.i./ha + cassia plant extract at 20-25 DAS (14.07), imazythapyre @ $56.25 \mathrm{~g}$ a.i./ha + parthenium plant extract at 20-25 DAS (14.02), imazythapyre @ $37.5 \mathrm{~g}$ a.i./ha + sorghum plant extract at 20-25 DAS (13.98), imazythapyre @ 56.25 g a.i./ha + eucalyptus leaf extract at 20-25 DAS (13.93), imazythapyre @ $37.5 \mathrm{~g}$ a.i./ha + cassia plant extract at 20-25 DAS (13.90), imazythapyre @ $37.5 \mathrm{~g}$ a.i./ha + parthenium plant extract at 20-25 DAS (13.83) and imazythapyre @ 37.5 g a.i./ha + eucalyptus leaf extract at 20-25 DAS (13.79), whereas unweeded check recorded significantly less number of primary branches (12.59).

\section{Number of secondary branches}

Weed management practices significantly influenced the number of secondary branches at harvest (Table 1). Weed free treatment recorded significantly higher number of secondary branches (12.43) and it was on par with imazythapyre @ $56.25 \mathrm{~g}$ a.i./ha + sorghum plant extract at 20-25 DAS (12.35), imazythapyre @ 56.25 g a.i./ha + cassia plant extract at 20-25 DAS (12.33), imazythapyre @ $56.25 \mathrm{~g}$ a.i./ha + parthenium plant extract at 20-25 DAS (12.28), imazythapyre @ $37.5 \mathrm{~g}$ a.i./ha + sorghum plant extract at 20-25 DAS (12.24), imazythapyre @ $56.25 \mathrm{~g}$ a.i./ha + eucalyptus leaf extract at 20-25 DAS (12.20), imazythapyre @ 37.5 g a.i./ha + cassia plant extract at 20-25 DAS (12.17), imazythapyre @ $37.5 \mathrm{~g}$ a.i./ha + parthenium plant extract at 20-25 DAS (12.11) and imazythapyre @ 37.5 $\mathrm{g}$ a.i./ha + eucalyptus leaf extract at 20-25 
DAS (12.08) and significantly lesser number of secondary branches (11.02) was seen in unweeded check, in pooled data.

Above results are in conformity with Arif et $a l$. , (2015) and he stated that allelopathy is an eco-friendly and organic weed management approach which may be used as a tool in controlling weeds.

Allelopathic water extracts of sorghum, sunflower and brassica applied at 25, 40 and 55 DAS each at 18 and $20 \mathrm{~L} \mathrm{ha}^{-1}$, significantly improved growth and yield parameters of wheat by weed management. Thus it's best to make use of plant extract or combination of herbicide and plant extract to control the weeds and improve the growth of crop.

\section{Seed yield per plant (g)}

Table 2 represents data related to seed yield per plant as influenced by plant extracts as weed management practice in Pigeonpea by allelopathy and varied significantly among weed management practices. Weed free treatment recorded significantly higher seed yield per plant $(63.2 \mathrm{~g})$ in pooled data, whereas it was on par with imazythapyre @ $56.25 \mathrm{~g}$ a.i./ha + sorghum plant extract at 2025 DAS (58.8 g), imazythapyre @ $56.25 \mathrm{~g}$ a.i./ha + cassia plant extract at 20-25 DAS (55.6 g), imazythapyre @ $56.25 \mathrm{~g}$ a.i./ha + parthenium plant extract at 20-25 DAS (54.4 g), imazythapyre @ 37.5 g a.i./ha + sorghum plant extract at 20-25 DAS (53.9 g), imazythapyre @ $56.25 \mathrm{~g}$ a.i./ha + eucalyptus leaf extract at 20-25 DAS $(53.4 \mathrm{~g})$, imazythapyre@37.5 g a.i./ha + cassia plant extract at 20-25 DAS (53.1 g), imazythapyre (a) $37.5 \mathrm{~g}$ a.i./ha + parthenium plant extract at 20-25 DAS (52.8 g) and imazythapyre @ 37.5 g a.i./ha + eucalyptus leaf extract at 20-25 DAS (52.3 g) and unweeded check showed significantly less seed yield per plant (26.2 g).

\section{0 seed weight (g)}

The data pertaining to 100 seed weight as influenced by allelopatheic effect of plant extracts as weed management practice in pigeonpea which varied significantly among different weed management practices is presented in Table 2. Significantly higher 100 seed weight was seen in weed free treatment $(9.72 \mathrm{~g})$, while imazythapyre @ $56.25 \mathrm{~g}$ a.i./ha + sorghum plant extract at 20-25 DAS $(9.59 \mathrm{~g})$, imazythapyre @ $56.25 \mathrm{~g}$ a.i./ha + cassia plant extract at 20-25 DAS (9.47 g), imazythapyre@ $56.25 \mathrm{~g}$ a.i./ha + parthenium plant extract at 20-25 DAS (9.45 g), imazythapyre @ 37.5 g a.i./ha + sorghum plant extract at 20-25 DAS (9.36 g), imazythapyre @ $56.25 \mathrm{~g}$ a.i./ha + eucalyptus leaf extract at 20-25 DAS (9.23 g), imazythapyre @ $37.5 \mathrm{~g}$ a.i./ha + cassia plant extract at 20-25 DAS (9.13 g), imazythapyre @ $37.5 \mathrm{~g}$ a.i./ha + parthenium plant extract at 20-25 DAS (9.09 g) and imazythapyre @ 37.5 g a.i./ha + eucalyptus leaf extract at 20-25 DAS (9.07 g) were on par with it and unweeded check (6.92 g) showed significantly lower 100 seed weight.

\section{Grain yield (kg ha-1)}

Grain yield as influenced by allelopatheic effect of plant extracts as weed management practice in pigeonpea is presented in the Table 3 and is clear that it varied significantly in different treatments. Significantly higher grain yield was seen in weed free treatment (2336 kg ha ${ }^{-1}$ ) and was on par with imazythapyre @ $56.25 \mathrm{~g}$ a.i./ha + sorghum plant extract at 20-25 DAS (2175 kg ha-1), imazythapyre @ $56.25 \mathrm{~g}$ a.i./ha + cassia plant extract at 20-25 DAS (2054 kg ha ${ }^{-1}$, imazythapyre@56.25 g a.i./ha + parthenium plant extract at 20-25 DAS (2014 kg ha-1), imazythapyre @37.5 g a.i./ha + sorghum plant extract at 20-25 DAS (1998 kg ha-1), imazythapyre @ $56.25 \mathrm{~g}$ a.i./ha + eucalyptus 
leaf extract at 20-25 DAS (1981 kg ha ${ }^{-1}$ ), imazythapyre@37.5 g a.i./ha + cassia plant extract at 20-25 DAS (1968 kg ha $\left.{ }^{-1}\right)$, imazythapyre @ 37.5 g a.i./ha + parthenium plant extract at 20-25 DAS (1954 kg ha ${ }^{-1}$ ) and imazythapyre @ 37.5 g a.i./ha + eucalyptus leaf extract at 20-25 DAS (1933 kg ha $\left.{ }^{-1}\right)$ and significantly lower grain yield was seen $(964$ $\mathrm{kg} \mathrm{ha}^{-1}$ ) in unweeded check in pooled data.

Extracts and residues of tested species might have the potential to be used for preemergence and post-emergence weed control (Nekonam et al., 2013). Application of reduced dose of herbicides mixed with allelopathic water extracts produced higher grain yield compared with the application of reduced dose of herbicides (Khan and Khan, 2012). Gilbert et al., (1999) reported that allelochemical facilitate nutrient solubilization and release nutrients from complex forms. Under low phosphorus (P) levels, plant release phosphatases which improve $\mathrm{P}$ availability through hydrolysis. Phenolics improve release and uptake of $\mathrm{P}$, Iron $(\mathrm{Fe})$ and other nutrients under their less availability. The basic function is the solublization of nutrients. They make nutrients more mobile and thus improve their uptake in plant body.

\section{Available soil nitrogen $\left(\mathrm{kg} \mathrm{ha}^{-1}\right)$}

Available soil nitrogen data as influenced by plant extracts as weed management practice in pigeonpea by allelopathy that varied significantly among different weed management practices is presented in Table 4 and Fig. 1. Significantly lower available soil nitrogen was seen in weed free treatment (221.95 kg ha ${ }^{-1}$ ) and was on par with imazythapyre@56.25 g a.i./ha + sorghum plant extract at 20-25 DAS (224.93 kg ha-1), imazythapyre @ $56.25 \mathrm{~g}$ a.i./ha + cassia plant extract at 20-25 DAS (227.71 kg ha-1), imazythapyre@ $56.25 \mathrm{~g}$ a.i./ha + parthenium plant extract at 20-25 DAS (228.30 kg ha $\left.{ }^{-1}\right)$, imazythapyre@ $37.5 \mathrm{~g}$ a.i./ha + sorghum plant extract at 20-25 DAS (229.20 kg ha'), imazythapyre @ $56.25 \mathrm{~g}$ a.i./ha + eucalyptus leaf extract at 20-25 DAS (229.73 kg ha'), imazythapyre@37.5 g a.i./ha + cassia plant extract at 20-25 DAS (231.46 kg ha-1), imazythapyre@37.5 g a.i./ha + parthenium plant extract at 20-25 DAS (232.29 $\mathrm{kg} \mathrm{ha}^{-1}$ ) and imazythapyre @ $37.5 \mathrm{~g}$ a.i./ha + eucalyptus leaf extract at 20-25 DAS (232.98 $\mathrm{kg} \mathrm{ha} \mathrm{h}^{-1}$ ) in pooled data and significantly higher available soil nitrogen was observed $\left(59.09 \mathrm{~kg} \mathrm{ha}^{-1}\right)$ in unweeded check.

\section{Available soil phosphorous ( $\mathrm{kg} \mathrm{ha}^{-1}$ )}

Available soil phosphorous data was influenced by allelopatheic effect of plant extracts as weed management practice in pigeonpea and showed that it varied significantly among different weed management practices (Table 5 and Fig. 1). Weed free treatment recorded significantly lower available soil phosphorous $(26.46 \mathrm{~kg}$ $\mathrm{ha}^{-1}$ ) in pooled data and was found to be on par with imazythapyre @ $56.25 \mathrm{~g}$ a.i./ha + sorghum plant extract at 20-25 DAS (27.05 $\mathrm{kg} \mathrm{ha}{ }^{-1}$ ), imazythapyre @ $56.25 \mathrm{~g}$ a.i./ha + cassia plant extract at 20-25 DAS $(27.69 \mathrm{~kg}$ $\mathrm{ha}^{-1}$ ), imazythapyre @ $56.25 \mathrm{~g}$ a.i./ha + parthenium plant extract at 20-25 DAS (28.15 $\mathrm{kg} \mathrm{ha}{ }^{-1}$ ), imazythapyre @ $37.5 \mathrm{~g}$ a.i./ha + sorghum plant extract at 20-25 DAS (28.21 $\mathrm{kg} \mathrm{ha}^{-1}$ ), imazythapyre @ $56.25 \mathrm{~g}$ a.i./ha + eucalyptus leaf extract at 20-25 DAS (28.53 $\mathrm{kg} \mathrm{ha}{ }^{-1}$ ), imazythapyre @ $37.5 \mathrm{~g}$ a.i./ha + cassia plant extract at 20-25 DAS $(28.72 \mathrm{~kg}$ $\left.\mathrm{ha}^{-1}\right)$, imazythapyre @ 37.5 g a.i./ha + parthenium plant extract at 20-25 DAS (28.91 $\mathrm{kg} \mathrm{ha}^{-1}$ ) and imazythapyre @ $37.5 \mathrm{~g}$ a.i./ha + eucalyptus leaf extract at 20-25 DAS (30.04 $\mathrm{kg} \mathrm{ha}^{-1}$ ) and significantly higher available soil phosphorous was witnessed in unweeded check (45.09 kg ha-1). 
Table.1 Primary branches and secondary branches as influenced by weed management in pigeonpea by allelopathy in pot culture experiment at harvest

\begin{tabular}{|c|c|c|c|c|c|c|}
\hline \multirow[t]{2}{*}{ Treatment } & \multicolumn{3}{|c|}{ Primary branches } & \multicolumn{3}{|c|}{ Secondary branches } \\
\hline & 2018 & 2019 & Pooled & 2018 & 2019 & Pooled \\
\hline $\begin{array}{l}\mathrm{T}_{1} \text { : Imazythapyre @ } 18.75 \mathrm{~g} \text { a.i./ha }+ \\
\text { Sorghum plant extract at 20-25 DAS }\end{array}$ & 13.02 & 13.88 & 13.45 & 11.41 & 12.16 & 11.78 \\
\hline $\begin{array}{l}\mathrm{T}_{2} \text { : Imazythapyre @ } 37.5 \mathrm{~g} \text { a.i./ha }+ \\
\text { Sorghum plant extract at 20-25 DAS }\end{array}$ & 13.59 & 14.37 & 13.98 & 11.90 & 12.59 & 12.24 \\
\hline $\begin{array}{l}\mathrm{T}_{3} \text { : Imazythapyre @ } 56.25 \mathrm{~g} \text { a.i./ha }+ \\
\text { Sorghum plant extract at 20-25 DAS }\end{array}$ & 13.70 & 14.50 & 14.10 & 12.00 & 12.70 & 12.35 \\
\hline $\begin{array}{l}T_{4} \text { : Only Sorghum plant extract at } 20-25 \\
\text { DAS }\end{array}$ & 12.61 & 13.43 & 13.02 & 11.05 & 11.76 & 11.41 \\
\hline $\begin{array}{l}\mathrm{T}_{5}: \text { Imazythapyre @ } 18.75 \mathrm{~g} \text { a.i./ha }+ \\
\text { Parthenium plant extract at 20-25 DAS }\end{array}$ & 12.77 & 13.58 & 13.18 & 11.18 & 11.90 & 11.54 \\
\hline $\begin{array}{l}\mathrm{T}_{6}: \text { Imazythapyre @ } 37.5 \mathrm{~g} \text { a.i./ha }+ \\
\text { Parthenium plant extract at } 20-25 \text { DAS }\end{array}$ & 13.44 & 14.22 & 13.83 & 11.77 & 12.46 & 12.11 \\
\hline $\begin{array}{l}\mathrm{T}_{7} \text { : Imazythapyre @ 56.25 g a.i./ha + } \\
\text { Parthenium plant extract at 20-25 DAS }\end{array}$ & 13.62 & 14.42 & 14.02 & 11.93 & 12.63 & 12.28 \\
\hline $\begin{array}{l}T_{8}: \text { Only Parthenium plant extract at } 20-25 \\
\text { DAS }\end{array}$ & 12.40 & 13.19 & 12.79 & 10.86 & 11.55 & 11.21 \\
\hline $\begin{array}{l}\mathrm{T}_{9}: \text { Imazythapyre @ } 18.75 \mathrm{~g} \text { a.i./ha }+ \\
\text { Eucalyptus leaf extract at 20-25 DAS }\end{array}$ & 12.67 & 13.48 & 13.08 & 11.10 & 11.81 & 11.45 \\
\hline $\begin{array}{l}\mathrm{T}_{10} \text { : Imazythapyre @ } 37.5 \mathrm{~g} \text { a.i./ha }+ \\
\text { Eucalyptus leaf extract at 20-25 DAS }\end{array}$ & 13.40 & 14.18 & 13.79 & 11.74 & 12.42 & 12.08 \\
\hline $\begin{array}{l}\mathrm{T}_{11} \text { : Imazythapyre @ } 56.25 \mathrm{~g} \text { a.i./ha + } \\
\text { Eucalyptus leaf extract at 20-25 DAS }\end{array}$ & 13.54 & 14.32 & 13.93 & 11.85 & 12.54 & 12.20 \\
\hline $\begin{array}{l}T_{12}: \text { Only Eucalyptus leaf extract at } 20-25 \\
\text { DAS }\end{array}$ & 12.52 & 13.32 & 12.92 & 10.97 & 11.67 & 11.32 \\
\hline $\begin{array}{l}\mathrm{T}_{13}: \text { Imazythapyre @ } 18.75 \mathrm{~g} \text { a.i./ha }+ \\
\text { Cassia plant extract at 20-25 DAS }\end{array}$ & 12.90 & 13.72 & 13.31 & 11.30 & 12.02 & 11.66 \\
\hline $\begin{array}{l}\mathrm{T}_{14}: \text { Imazythapyre @ } 37.5 \mathrm{~g} \text { a.i./ha }+ \\
\text { Cassia plant extract at 20-25 DAS }\end{array}$ & 13.51 & 14.29 & 13.90 & 11.83 & 12.52 & 12.17 \\
\hline $\begin{array}{l}\mathrm{T}_{15}: \text { Imazythapyre @ } 56.25 \mathrm{~g} \text { a.i./ha }+ \\
\text { Cassia plant extract at 20-25 DAS }\end{array}$ & 13.68 & 14.47 & 14.07 & 11.98 & 12.68 & 12.33 \\
\hline $\begin{array}{l}T_{16}: \text { Only Cassia plant extract at } 20-25 \\
\text { DAS }\end{array}$ & 12.46 & 13.25 & 12.85 & 10.91 & 11.61 & 11.26 \\
\hline$T_{17}:$ Weed free & 13.79 & 14.59 & 14.19 & 12.08 & 12.78 & 12.43 \\
\hline $\mathrm{T}_{18}$ : Unweeded check & 12.20 & 12.98 & 12.59 & 10.68 & 11.37 & 11.02 \\
\hline S.Em \pm & 0.20 & 0.17 & 0.19 & 0.19 & 0.17 & 0.18 \\
\hline C.D. at $5 \%$ & 0.79 & 0.67 & 0.72 & 0.73 & 0.67 & 0.69 \\
\hline
\end{tabular}


Table.2 Seed yield per plant (g) and 100 seed weight $(\mathrm{g})$ as influenced by weed management in pigeonpea by allelopathy in pot culture experiment

\begin{tabular}{|c|c|c|c|c|c|c|}
\hline \multirow[t]{2}{*}{ Treatment } & \multicolumn{3}{|c|}{ Seed yield per plant (g) } & \multicolumn{3}{|c|}{100 seed weight (g) } \\
\hline & 2018 & 2019 & Pooled & 2018 & 2019 & Pooled \\
\hline $\begin{array}{l}\mathrm{T}_{1} \text { : Imazythapyre @ } 18.75 \mathrm{~g} \text { a.i. } / \mathrm{ha}+ \\
\text { Sorghum plant extract at 20-25 DAS }\end{array}$ & 41.3 & 47.4 & 44.4 & 7.89 & 8.17 & 8.03 \\
\hline $\begin{array}{l}\mathrm{T}_{2} \text { : Imazythapyre @ } 37.5 \mathrm{~g} \text { a.i./ha }+ \\
\text { Sorghum plant extract at 20-25 DAS }\end{array}$ & 50.3 & 57.5 & 53.9 & 9.20 & 9.52 & 9.36 \\
\hline $\begin{array}{l}\mathrm{T}_{3} \text { : Imazythapyre @ } 56.25 \mathrm{~g} \text { a.i./ha }+ \\
\text { Sorghum plant extract at 20-25 DAS }\end{array}$ & 55.5 & 62.0 & 58.8 & 9.43 & 9.76 & 9.59 \\
\hline $\mathrm{T}_{4}$ : Only Sorghum plant extract at 20-25 DAS & 27.1 & 38.0 & 32.6 & 7.39 & 7.65 & 7.52 \\
\hline $\begin{array}{l}\mathrm{T}_{5}: \text { Imazythapyre @ } 18.75 \mathrm{~g} \text { a.i./ha }+ \\
\text { Parthenium plant extract at 20-25 DAS }\end{array}$ & 36.6 & 46.0 & 41.3 & 7.36 & 7.62 & 7.49 \\
\hline $\begin{array}{l}\mathrm{T}_{6}: \text { Imazythapyre @ } 37.5 \mathrm{~g} \text { a.i./ha }+ \\
\text { Parthenium plant extract at20-25 DAS }\end{array}$ & 49.3 & 56.3 & 52.8 & 8.93 & 9.25 & 9.09 \\
\hline $\begin{array}{l}\mathrm{T}_{7}: \text { Imazythapyre @ } 56.25 \mathrm{~g} \text { a.i./ha }+ \\
\text { Parthenium plant extract at } 20-25 \text { DAS }\end{array}$ & 50.7 & 58.1 & 54.4 & 9.29 & 9.61 & 9.45 \\
\hline $\begin{array}{l}T_{8}: \text { Only Parthenium plant extract at } 20-25 \\
\text { DAS }\end{array}$ & 24.8 & 36.1 & 30.5 & 6.90 & 7.14 & 7.02 \\
\hline $\begin{array}{l}\mathrm{T}_{9} \text { : Imazythapyre @ } 18.75 \mathrm{~g} \text { a.i./ha }+ \\
\text { Eucalyptus leaf extract at 20-25 DAS }\end{array}$ & 35.4 & 45.1 & 40.3 & 7.62 & 7.89 & 7.75 \\
\hline $\begin{array}{l}\mathrm{T}_{10} \text { : Imazythapyre @ } 37.5 \mathrm{~g} \text { a.i./ha }+ \\
\text { Eucalyptus leaf extract at 20-25 DAS }\end{array}$ & 48.4 & 56.2 & 52.3 & 8.90 & 9.24 & 9.07 \\
\hline $\begin{array}{l}\mathrm{T}_{11} \text { : Imazythapyre @ } 56.25 \mathrm{~g} \text { a.i./ha }+ \\
\text { Eucalyptus leaf extract at 20-25 DAS }\end{array}$ & 49.6 & 57.2 & 53.4 & 9.07 & 9.39 & 9.23 \\
\hline $\begin{array}{l}T_{12} \text { : Only Eucalyptus leaf extract at } 20-25 \\
\text { DAS }\end{array}$ & 27.1 & 37.9 & 32.5 & 7.19 & 7.44 & 7.32 \\
\hline $\begin{array}{l}\mathrm{T}_{13} \text { : Imazythapyre @ } 18.75 \mathrm{~g} \text { a.i./ha }+ \\
\text { Cassia plant extract at } 20-25 \mathrm{DAS}\end{array}$ & 37.8 & 47.0 & 42.4 & 7.80 & 8.07 & 7.94 \\
\hline $\begin{array}{l}\mathrm{T}_{14} \text { : Imazythapyre @ } 37.5 \mathrm{~g} \text { a.i./ha }+ \\
\text { Cassia plant extract at 20-25 DAS }\end{array}$ & 49.5 & 56.7 & 53.1 & 8.97 & 9.28 & 9.13 \\
\hline $\begin{array}{l}\mathrm{T}_{15} \text { : Imazythapyre @ } 56.25 \mathrm{~g} \text { a.i./ha }+ \\
\text { Cassia plant extract at } 20-25 \mathrm{DAS}\end{array}$ & 51.9 & 59.2 & 55.6 & 9.30 & 9.63 & 9.47 \\
\hline $\begin{array}{l}T_{16}: \text { Only Cassia plant extract at } \mathbf{2 0 - 2 5} \\
\text { DAS }\end{array}$ & 26.0 & 37.2 & 31.6 & 7.10 & 7.35 & 7.22 \\
\hline$T_{17}:$ Weed free & 60.2 & 66.2 & 63.2 & 9.56 & 9.89 & 9.72 \\
\hline$T_{18}:$ Unweeded check & 20.1 & 32.2 & 26.2 & 6.80 & 7.04 & 6.92 \\
\hline S.Em \pm & 3.5 & 4.1 & 3.0 & 0.17 & 0.17 & 0.17 \\
\hline C.D. at $5 \%$ & 13.4 & 15.6 & 11.5 & 0.65 & 0.65 & 0.65 \\
\hline
\end{tabular}


Table.3 Grain yield $\left(\mathrm{kg} \mathrm{ha}^{-1}\right)$ as influenced by weed management in pigeonpea by allelopathy in pot culture experiment

\begin{tabular}{|c|c|c|c|}
\hline \multirow[t]{2}{*}{ Treatment } & \multicolumn{3}{|c|}{ Grain yield $\left(\mathrm{kg} \mathrm{ha}^{-1}\right)$} \\
\hline & 2018 & 2019 & Pooled \\
\hline $\begin{array}{l}\mathrm{T}_{1} \text { : Imazythapyre @ } 18.75 \mathrm{~g} \text { a.i./ha + Sorghum } \\
\text { plant extract at 20-25 DAS }\end{array}$ & 1529 & 1752 & 1641 \\
\hline $\begin{array}{l}\mathrm{T}_{2} \text { : Imazythapyre @ } 37.5 \mathrm{~g} \text { a.i./ha + Sorghum plant } \\
\text { extract at 20-25 DAS }\end{array}$ & 1869 & 2126 & 1998 \\
\hline $\begin{array}{l}\mathrm{T}_{3}: \text { Imazythapyre @ } 56.25 \mathrm{~g} \text { a.i./ha + Sorghum } \\
\text { plant extract at 20-25 DAS }\end{array}$ & 2054 & 2296 & 2175 \\
\hline$T_{4}$ : Only Sorghum plant extract at 20-25 DAS & 1005 & 1407 & 1206 \\
\hline $\begin{array}{l}\mathrm{T}_{5} \text { : Imazythapyre @ } 18.75 \mathrm{~g} \text { a.i./ha + Parthenium } \\
\text { plant extract at 20-25 DAS }\end{array}$ & 1355 & 1703 & 1529 \\
\hline $\begin{array}{l}\mathrm{T}_{6}: \text { Imazythapyre @ } 37.5 \mathrm{~g} \text { a.i./ha + Parthenium } \\
\text { plant extract at 20-25 DAS }\end{array}$ & 1827 & 2081 & 1954 \\
\hline $\begin{array}{l}\mathrm{T}_{7}: \text { Imazythapyre @ } 56.25 \mathrm{~g} \text { a.i./ha + Parthenium } \\
\text { plant extract at 20-25 DAS }\end{array}$ & 1879 & 2148 & 2014 \\
\hline $\mathrm{T}_{8}$ : Only Parthenium plant extract at 20-25 DAS & 918 & 1333 & 1126 \\
\hline $\begin{array}{l}\text { T9: Imazythapyre @ } 18.75 \text { g a.i./ha + Eucalyptus } \\
\text { leaf extract at 20-25 DAS }\end{array}$ & 1311 & 1666 & 1489 \\
\hline $\begin{array}{l}\mathrm{T}_{10} \text { : Imazythapyre @ } 37.5 \mathrm{~g} \text { a.i./ha + Eucalyptus } \\
\text { leaf extract at 20-25 DAS }\end{array}$ & 1792 & 2074 & 1933 \\
\hline $\begin{array}{l}\mathrm{T}_{11}: \text { Imazythapyre @ } 56.25 \mathrm{~g} \text { a.i./ha + Eucalyptus } \\
\text { leaf extract at 20-25 DAS }\end{array}$ & 1850 & 2111 & 1981 \\
\hline $\mathrm{T}_{12}:$ Only Eucalyptus leaf extract at 20-25 DAS & 1005 & 1400 & 1203 \\
\hline $\begin{array}{l}\mathrm{T}_{13} \text { : Imazythapyre @ } 18.75 \mathrm{~g} \text { a.i./ha + Cassia plant } \\
\text { extract at 20-25 DAS }\end{array}$ & 1398 & 1740 & 1569 \\
\hline $\begin{array}{l}\mathrm{T}_{14}: \text { Imazythapyre @ } 37.5 \mathrm{~g} \text { a.i./ha + Cassia plant } \\
\text { extract at 20-25 DAS }\end{array}$ & 1835 & 2100 & 1968 \\
\hline $\begin{array}{l}\mathrm{T}_{15}: \text { Imazythapyre @ } 56.25 \text { g a.i./ha + Cassia plant } \\
\text { extract at 20-25 DAS }\end{array}$ & 1923 & 2185 & 2054 \\
\hline $\mathrm{T}_{16}$ : Only Cassia plant extract at 20-25 DAS & 961 & 1370 & 1166 \\
\hline$T_{17}:$ Weed free & 2228 & 2444 & 2336 \\
\hline$T_{18}:$ Unweeded check & 743 & 1185 & 964 \\
\hline S.Em \pm & 129 & 150 & 111 \\
\hline C.D. at $5 \%$ & 494 & 577 & 428 \\
\hline
\end{tabular}


Table.4 Available soil nitrogen $\left(\mathrm{kg} \mathrm{ha}^{-1}\right)$ status after harvest as influenced by weed management in pigeonpea by allelopathy in pot culture experiment

\begin{tabular}{|c|c|c|c|}
\hline \multirow[t]{2}{*}{ Treatment } & \multicolumn{3}{|c|}{ Nitrogen $\left(\mathrm{kg} \mathrm{ha}^{-1}\right)$} \\
\hline & 2018 & 2019 & Pooled \\
\hline $\begin{array}{l}\mathrm{T}_{1} \text { : Imazythapyre @ } 18.75 \mathrm{~g} \text { a.i./ha + Sorghum } \\
\text { plant extract at 20-25 DAS }\end{array}$ & 237.80 & 234.70 & 236.25 \\
\hline $\begin{array}{l}\mathrm{T}_{2} \text { : Imazythapyre @ } 37.5 \mathrm{~g} \text { a.i./ha + Sorghum plant } \\
\text { extract at 20-25 DAS }\end{array}$ & 230.70 & 227.70 & 229.20 \\
\hline $\begin{array}{l}\mathrm{T}_{3} \text { : Imazythapyre @ } 56.25 \mathrm{~g} \text { a.i./ha + Sorghum } \\
\text { plant extract at 20-25 DAS }\end{array}$ & 226.40 & 223.46 & 224.93 \\
\hline$T_{4}$ : Only Sorghum plant extract at 20-25 DAS & 252.30 & 249.02 & 250.66 \\
\hline $\begin{array}{l}\text { T}_{5}: \text { Imazythapyre @ } 18.75 \text { g a.i./ha + Parthenium } \\
\text { plant extract at 20-25 DAS }\end{array}$ & 242.88 & 239.72 & 241.30 \\
\hline $\begin{array}{l}\mathrm{T}_{6}: \text { Imazythapyre @ } 37.5 \mathrm{~g} \text { a.i./ha + Parthenium } \\
\text { plant extract at 20-25 DAS }\end{array}$ & 233.80 & 230.79 & 232.29 \\
\hline $\begin{array}{l}\mathrm{T}_{7}: \text { Imazythapyre @ } 56.25 \mathrm{~g} \text { a.i./ha + Parthenium } \\
\text { plant extract at 20-25 DAS }\end{array}$ & 229.80 & 226.80 & 228.30 \\
\hline$T_{8}$ : Only Parthenium plant extract at 20-25 DAS & 255.40 & 252.08 & 253.74 \\
\hline $\begin{array}{l}\text { T9: Imazythapyre @ } 18.75 \text { g a.i./ha + Eucalyptus } \\
\text { leaf extract at 20-25 DAS }\end{array}$ & 247.50 & 244.28 & 245.89 \\
\hline $\begin{array}{l}\mathrm{T}_{10} \text { : Imazythapyre @ } 37.5 \mathrm{~g} \text { a.i./ha + Eucalyptus } \\
\text { leaf extract at 20-25 DAS }\end{array}$ & 234.50 & 231.45 & 232.98 \\
\hline $\begin{array}{l}\mathrm{T}_{11} \text { : Imazythapyre @ } 56.25 \mathrm{~g} \text { a.i./ha + Eucalyptus } \\
\text { leaf extract at 20-25 DAS }\end{array}$ & 231.23 & 228.22 & 229.73 \\
\hline $\mathrm{T}_{12}$ : Only Eucalyptus leaf extract at 20-25 DAS & 251.10 & 247.84 & 249.47 \\
\hline $\begin{array}{l}\mathrm{T}_{13} \text { : Imazythapyre @ } 18.75 \mathrm{~g} \text { a.i./ha + Cassia plant } \\
\text { extract at 20-25 DAS }\end{array}$ & 240.72 & 237.59 & 239.16 \\
\hline $\begin{array}{l}\text { T}_{14} \text { : Imazythapyre @ } 37.5 \mathrm{~g} \text { a.i./ha + Cassia plant } \\
\text { extract at 20-25 DAS }\end{array}$ & 232.97 & 229.94 & 231.46 \\
\hline $\begin{array}{l}\mathrm{T}_{15}: \text { Imazythapyre @ } 56.25 \text { g a.i./ha + Cassia plant } \\
\text { extract at 20-25 DAS }\end{array}$ & 229.20 & 226.22 & 227.71 \\
\hline$T_{16}:$ Only Cassia plant extract at 20-25 DAS & 253.50 & 250.20 & 251.85 \\
\hline$T_{17}:$ Weed free & 223.40 & 220.50 & 221.95 \\
\hline $\mathbf{T}_{18}:$ Unweeded check & 257.88 & 254.53 & 256.20 \\
\hline S.Em \pm & 3.45 & 3.00 & 2.99 \\
\hline C.D. at $5 \%$ & 13.28 & 11.53 & 11.48 \\
\hline
\end{tabular}


Table.5 Available soil phosphorous $\left(\mathrm{kg} \mathrm{ha}^{-1}\right)$ status after harvest as influenced by weed management in pigeonpea by allelopathy in pot culture experiment

\begin{tabular}{|c|c|c|c|}
\hline \multirow[t]{2}{*}{ Treatment } & \multicolumn{3}{|c|}{ Phosphorous (kg ha $\left.{ }^{-1}\right)$} \\
\hline & 2018 & 2019 & Pooled \\
\hline $\begin{array}{l}\mathrm{T}_{1} \text { : Imazythapyre @ } 18.75 \mathrm{~g} \text { a.i./ha + Sorghum } \\
\text { plant extract at 20-25 DAS }\end{array}$ & 31.80 & 30.51 & 31.15 \\
\hline $\begin{array}{l}\mathrm{T}_{2} \text { : Imazythapyre @ } 37.5 \mathrm{~g} \text { a.i./ha + Sorghum plant } \\
\text { extract at 20-25 DAS }\end{array}$ & 28.80 & 27.62 & 28.21 \\
\hline $\begin{array}{l}\mathrm{T}_{3}: \text { Imazythapyre @ } 56.25 \mathrm{~g} \text { a.i./ha + Sorghum } \\
\text { plant extract at 20-25 DAS }\end{array}$ & 27.60 & 26.50 & 27.05 \\
\hline $\mathrm{T}_{4}$ : Only Sorghum plant extract at 20-25 DAS & 37.84 & 36.33 & 37.08 \\
\hline $\begin{array}{l}\mathrm{T}_{5} \text { : Imazythapyre @ } 18.75 \mathrm{~g} \text { a.i./ha + Parthenium } \\
\text { plant extract at 20-25 DAS }\end{array}$ & 33.21 & 31.90 & 32.56 \\
\hline $\begin{array}{l}\mathrm{T}_{6}: \text { Imazythapyre @ } 37.5 \mathrm{~g} \text { a.i./ha + Parthenium } \\
\text { plant extract at 20-25 DAS }\end{array}$ & 29.50 & 28.32 & 28.91 \\
\hline $\begin{array}{l}\mathrm{T}_{7}: \text { Imazythapyre @ } 56.25 \mathrm{~g} \text { a.i./ha + Parthenium } \\
\text { plant extract at 20-25 DAS }\end{array}$ & 28.72 & 27.57 & 28.15 \\
\hline $\mathrm{T}_{8}$ : Only Parthenium plant extract at 20-25 DAS & 41.18 & 39.50 & 40.34 \\
\hline $\begin{array}{l}\text { T9: Imazythapyre @ } 18.75 \text { g a.i./ha + Eucalyptus } \\
\text { leaf extract at 20-25 DAS }\end{array}$ & 34.50 & 33.12 & 33.81 \\
\hline $\begin{array}{l}\mathrm{T}_{10} \text { : Imazythapyre @ } 37.5 \mathrm{~g} \text { a.i./ha + Eucalyptus } \\
\text { leaf extract at 20-25 DAS }\end{array}$ & 30.65 & 29.42 & 30.04 \\
\hline $\begin{array}{l}\mathrm{T}_{11}: \text { Imazythapyre @ } 56.25 \mathrm{~g} \text { a.i./ha + Eucalyptus } \\
\text { leaf extract at 20-25 DAS }\end{array}$ & 29.11 & 27.95 & 28.53 \\
\hline$T_{12}:$ Only Eucalyptus leaf extract at 20-25 DAS & 37.93 & 36.41 & 37.17 \\
\hline $\begin{array}{l}\mathrm{T}_{13} \text { : Imazythapyre @ } 18.75 \mathrm{~g} \text { a.i./ha + Cassia plant } \\
\text { extract at 20-25 DAS }\end{array}$ & 32.10 & 30.82 & 31.46 \\
\hline $\begin{array}{l}\mathrm{T}_{14}: \text { Imazythapyre @ } 37.5 \mathrm{~g} \text { a.i./ha + Cassia plant } \\
\text { extract at 20-25 DAS }\end{array}$ & 29.31 & 28.14 & 28.72 \\
\hline $\begin{array}{l}\mathrm{T}_{15}: \text { Imazythapyre @ } 56.25 \text { g a.i./ha + Cassia plant } \\
\text { extract at 20-25 DAS }\end{array}$ & 28.25 & 27.12 & 27.69 \\
\hline $\mathrm{T}_{16}$ : Only Cassia plant extract at 20-25 DAS & 39.80 & 38.21 & 39.00 \\
\hline$T_{17}:$ Weed free & 27.00 & 25.92 & 26.46 \\
\hline$T_{18}:$ Unweeded check & 42.30 & 47.88 & 45.09 \\
\hline S.Em \pm & 1.08 & 1.18 & 0.97 \\
\hline C.D. at $5 \%$ & 4.16 & 4.53 & 3.71 \\
\hline
\end{tabular}


Table.6 Available soil potassium $\left(\mathrm{kg} \mathrm{ha}^{-1}\right)$ status after harvest as influenced by weed management in pigeonpea by allelopathy in pot culture experiment

\begin{tabular}{|c|c|c|c|}
\hline \multirow[t]{2}{*}{ Treatment } & \multicolumn{3}{|c|}{ Potassium $\left(\mathrm{kg} \mathrm{ha}^{-1}\right)$} \\
\hline & 2018 & 2019 & Pooled \\
\hline $\begin{array}{l}\mathrm{T}_{1} \text { : Imazythapyre @ } 18.75 \mathrm{~g} \text { a.i./ha + Sorghum } \\
\text { plant extract at 20-25 DAS }\end{array}$ & 368.50 & 361.11 & 364.81 \\
\hline $\begin{array}{l}\mathrm{T}_{2} \text { : Imazythapyre @ } 37.5 \mathrm{~g} \text { a.i./ha + Sorghum plant } \\
\text { extract at 20-25 DAS }\end{array}$ & 361.80 & 354.56 & 358.18 \\
\hline $\begin{array}{l}\mathrm{T}_{3}: \text { Imazythapyre @ } 56.25 \mathrm{~g} \text { a.i./ha + Sorghum } \\
\text { plant extract at 20-25 DAS }\end{array}$ & 359.11 & 351.94 & 355.52 \\
\hline $\mathrm{T}_{4}:$ Only Sorghum plant extract at 20-25 DAS & 375.66 & 368.15 & 371.90 \\
\hline $\begin{array}{l}\mathrm{T}_{5}: \text { Imazythapyre @ } 18.75 \mathrm{~g} \text { a.i./ha + Parthenium } \\
\text { plant extract at 20-25 DAS }\end{array}$ & 370.80 & 363.38 & 367.09 \\
\hline $\begin{array}{l}\text { T}_{6}: \text { Imazythapyre @ } 37.5 \mathrm{~g} \text { a.i./ha + Parthenium } \\
\text { plant extract at 20-25 DAS }\end{array}$ & 363.50 & 356.23 & 359.87 \\
\hline $\begin{array}{l}\mathrm{T}_{7}: \text { Imazythapyre @ } 56.25 \mathrm{~g} \text { a.i./ha + Parthenium } \\
\text { plant extract at 20-25 DAS }\end{array}$ & 361.30 & 354.07 & 357.69 \\
\hline$T_{8}$ : Only Parthenium plant extract at 20-25 DAS & 378.50 & 370.93 & 374.72 \\
\hline $\begin{array}{l}\text { T9: Imazythapyre @ } 18.75 \text { g a.i./ha + Eucalyptus } \\
\text { leaf extract at 20-25 DAS }\end{array}$ & 372.88 & 365.42 & 369.15 \\
\hline $\begin{array}{l}\mathrm{T}_{10}: \text { Imazythapyre @ } 37.5 \mathrm{~g} \text { a.i./ha + Eucalyptus } \\
\text { leaf extract at 20-25 DAS }\end{array}$ & 365.20 & 357.90 & 361.55 \\
\hline $\begin{array}{l}\mathrm{T}_{11} \text { : Imazythapyre @ } 56.25 \mathrm{~g} \text { a.i./ha + Eucalyptus } \\
\text { leaf extract at 20-25 DAS }\end{array}$ & 362.58 & 355.33 & 358.95 \\
\hline$T_{12}:$ Only Eucalyptus leaf extract at 20-25 DAS & 376.23 & 368.71 & 372.47 \\
\hline $\begin{array}{l}\mathrm{T}_{13}: \text { Imazythapyre @ } 18.75 \mathrm{~g} \text { a.i./ha + Cassia plant } \\
\text { extract at 20-25 DAS }\end{array}$ & 369.80 & 362.40 & 366.10 \\
\hline $\begin{array}{l}\mathrm{T}_{14}: \text { Imazythapyre @ } 37.5 \mathrm{~g} \text { a.i./ha + Cassia plant } \\
\text { extract at 20-25 DAS }\end{array}$ & 362.77 & 355.51 & 359.14 \\
\hline $\begin{array}{l}\mathrm{T}_{15}: \text { Imazythapyre @ } 56.25 \mathrm{~g} \text { a.i./ha + Cassia plant } \\
\text { extract at } 20-25 \text { DAS }\end{array}$ & 360.20 & 353.00 & 356.60 \\
\hline $\mathrm{T}_{16}:$ Only Cassia plant extract at 20-25 DAS & 377.60 & 370.05 & 373.82 \\
\hline$T_{17}:$ Weed free & 357.40 & 350.25 & 353.83 \\
\hline$T_{18}:$ Unweeded check & 380.50 & 372.89 & 376.70 \\
\hline S.Em \pm & 2.30 & 2.16 & 2.11 \\
\hline C.D. at $5 \%$ & 8.84 & 8.32 & 8.12 \\
\hline
\end{tabular}


Fig.1 Available soil nutrient status $\left(\mathrm{kg} \mathrm{ha}^{-1}\right)$ after harvest as influenced by weed management in pigeonpea by allelopathy in pot culture experiment

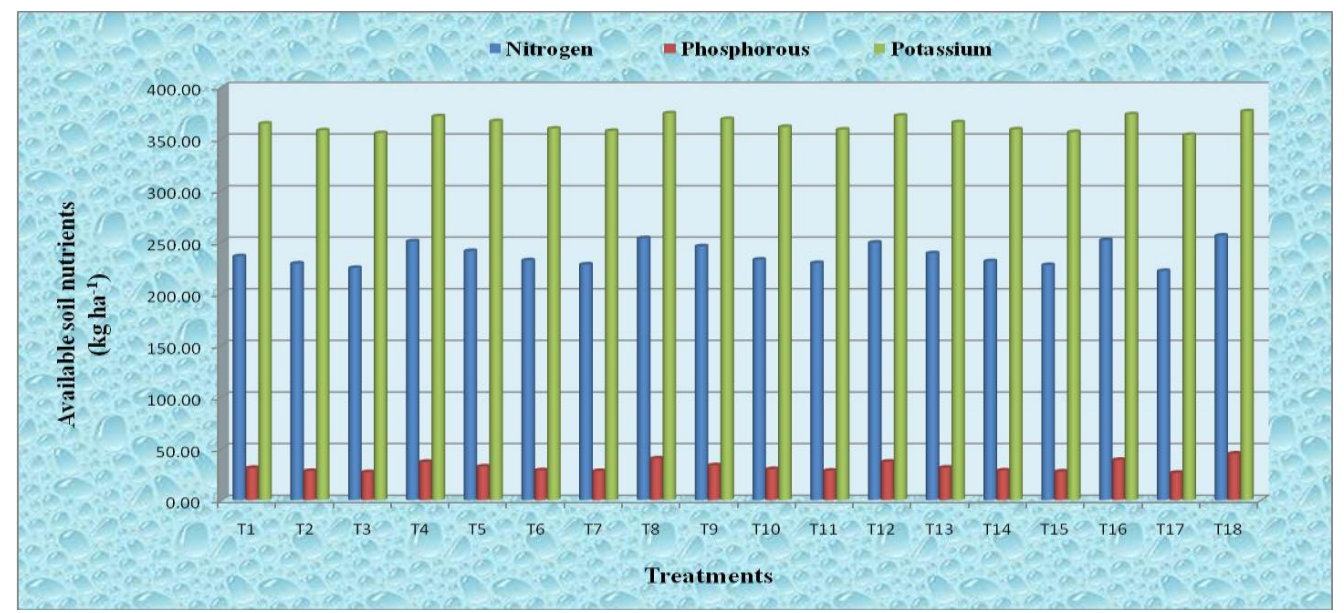

\section{Available soil potassium $\left(\mathrm{kg} \mathrm{ha}^{-1}\right)$}

Available soil potassium data was influenced by allelopatheic effect of plant extracts as weed management practice in Pigeonpea and showed that it varied significantly among different weed management practices (Table 6 and Fig. 1). In pooled data, imazythapyre @ $56.25 \mathrm{~g}$ a.i./ha + cassia plant extract at 20-25 DAS (355.52 Kg ha ${ }^{-1}$ ), imazythapyre @ 56.25 g a.i./ha + parthenium plant extract at 20-25 DAS (356.60 $\left.\mathrm{kg} \mathrm{ha}^{-1}\right)$, imazythapyre @ $37.5 \mathrm{~g}$ a.i./ha + sorghum plant extract at 20-25 DAS (357.69 kg ha-1), imazythapyre @ $56.25 \mathrm{~g}$ a.i./ha + eucalyptus leaf extract at 20-25 DAS (358.18 kg ha $\mathrm{kg}^{-1}$, imazythapyre @ $37.5 \mathrm{~g}$ a.i./ha + cassia plant extract at 20-25 DAS (358.95 kg ha ${ }^{-1}$ ), imazythapyre @ $37.5 \mathrm{~g}$ a.i./ha + parthenium plant extract at 20-25 DAS (359.14 kg ha ${ }^{-1}$ ), imazythapyre @ $37.5 \mathrm{~g}$ a.i./ha + eucalyptus leaf extract at 20-25 DAS (359.87 $\mathrm{kg} \mathrm{ha}^{-1}$ ) and imazythapyre @ $56.25 \mathrm{~g}$ a.i./ha + sorghum plant extract at 20-25 DAS $\left(361.55 \mathrm{~kg} \mathrm{ha}^{-1}\right)$ were found on par with weed free treatment $\left(353.83 \mathrm{~kg} \mathrm{ha}^{-1}\right)$ that recorded significantly lower available soil potassium and unweeded check treatment remarked significantly higher available soil potassium (376.70 kg ha $\left.{ }^{-1}\right)$.
Weed management practices significantly influenced the available nutrients in soil $(\mathrm{N}, \mathrm{P}$ and $\mathrm{K}$ ) among all the treatments after harvest of pigeonpea. Imazythapyre @ $56.25 \mathrm{~g}$ a.i./ha + sorghum plant extract at 20-25 DAS, imazythapyre @ $56.25 \mathrm{~g}$ a.i./ha + cassia plant extract at 20-25 DAS, imazythapyre @ 56.25 g a.i./ha + parthenium plant extract at 20-25 DAS, imazythapyre @ $37.5 \mathrm{~g}$ a.i./ha + sorghum plant extract at 20-25 DAS, imazythapyre@ $56.25 \mathrm{~g}$ a.i./ha + eucalyptus leaf extract at 20-25 DAS, imazythapyre @ $37.5 \mathrm{~g}$ a.i./ha + cassia plant extract at 20-25 DAS, imazythapyre @ $37.5 \mathrm{~g}$ a.i./ha + parthenium plant extract at 20-25 DAS and imazythapyre @ $37.5 \mathrm{~g}$ a.i./ha + eucalyptus leaf extract at 20-25 DAS recorded significantly lower available nitrogen, phosphorus and potassium compared to rest of the treatments which might be due better growth of crop in these treatments and therefore good vegetative and reproductive growth helped crop to use available nutrients in the soil.

Allelochemicals, upon release into the rhizosphere may influence nutrient movement, availability and uptake by plants. Changes in microbial activities and nutrient dynamics on addition of allelochemicals to 
the soil have been reported (Karmarkar and Tabatabai 1991). Usually, allelochemicals are first perceived by the receiver plant's roots which may then affect nutrient uptake and these compounds may restrict or improve the nutrients mobility to plants ( $\mathrm{Yu}$ and Matsui 1997). Gilbert et al., (1999) reported that allelochemical facilitate nutrient solublization and release from complex forms. Under low phosphorus (P) levels plant release phosphatases which improve $\mathrm{P}$ availability through hydrolysis. Phenolics improve release and uptake of $\mathrm{P}$, Iron $(\mathrm{Fe})$ and other nutrients under their less availability. The basic function is the solublization of nutrients. They make nutrients more mobile and thus improve their uptake in plant body. Dalipu (2001) studied the effect of different weed species on leaf total chlorophyll content and leaf nitrate reductase (NR) activity of rice plant. They reported that both the weeds Cynadon dactylon and Cyperus rotundus at low concentration (1:20) increased the total chlorophyll content, nitrate reductase (NR) activity of rice plant and soil available $\mathrm{N}, \mathrm{P}$ and $\mathrm{K}$ were low at 25 and 50 DAS which might be due to growth promotion property of allelopathic water extracts of these weeds at low concentration.

Plant extracts of Sorghum, Parthenium, Cassia and Eucalyptus with imazythapyre herbicide at $50 \%$ and $75 \%$ help to get good control of weeds in pigeonpea. Thereby help to improve the growth of pigeonpea indirectly and through releasing allelochemicals directly into crop rhizosphere. Hence allelochemicals can be used with herbicide which will help to reduce usage of herbicide by $50 \%$ and dependency on herbicides can be reduced, through which ill effects of herbicides on soil can be avoided. Soil fertility and productivity can be maintained for longer period through which crop production can become more productive, sustainable and economical both directly and indirectly.

\section{References}

Anonymous, 2019, https://www.indiastat.com/table/agricult ure-data/2/arha-tur/19566/17 337/data.aspx.

Arif, M., Cheema, Z. A., Khaliq, A. and Hassan, A., 2015, Organic weed management in wheat through allelopathy. Int. J. Agric. Biol., 17: 127134.

Dalipu, K., 2001, Allelopathic effect of weeds on physio-chemical properties of rice and nutrient status of soil. Ecol. Env. and Cons., 7(1): 79-85.

Gilbert, G. A., Knight, J. D., Allan, D. L. and Vance, C. P., 1999, Acid phosphatase activity in phosphorus deficient white lupin roots. Plant Cell Environ., 22: 801-810.

Jackson, M. L., 1973, Soil Chemical Analysis. Oxford IBH Publishing House, Bombay. p. 38.

Karmarkar, S. V. and Tabatabai, M. A., 1991, Effects of biotechnology byproducts and organic acids on nitrification in soils. Biol. Fertil. Soils., 12: 165-169.

Khan, R. and Khan, A. M., 2012, Weed control efficiency of bioherbicides and their impact on grain yield of wheat (Triticum aestivum L.). European J. Appl. Sci., 4(5): 216-219.

Nekonam, M. S., Jamshid, R., Hasan, K., Bahram, S., Hajar, A. and Frouzan, B., 2013, Assessment of some medicinal plants for their allelopathic potential against redroot pigweed (Amaranthus retroflexus). J. Plant Prot. Res., 54(1): 90-95.

Panse, V. G. and Sukhatme, P. V., 1967, Statistical Methods for Agricultural Workers, Indian Council of Agricultural Research, New Delhi.

Pooniya, V., Choudhary, A. K., Dass, A., Bana, R. S., Rana, K. S., Rana, D. S., Tyagi, V. K. and Puniya, M. M., 2015, 
Improved crop management practices for sustainable pulse production: An Indian perspective. Indian J. Agric. Sci., 85(6): 747-758.

Subbaiah, B. Y. and Asija, G. L., 1956, A rapid procedure for the estimation of available nitrogen in soils. Curr. Sci., 25: 259-260.

Talnikar, A. S., Kadam, D. R., Karande, D. R. and Jogdand, P. B., 2008, Integrated weed management in pigeonpea [Cajanus cajan (L.) Millsp.]. Int. J. Agric. Sci., 4: 363-370.

Yu, J. Q. and Matsui, Y., 1997, Effects of root exudates of cucumber (Cucumis sativus) and allelochemicals on ion uptake by cucumber seedlings. J. Chem. Ecol., 23: $817-827$.

\section{How to cite this article:}

Shridhara, B. Nagoli, B. M. Dodamani, M. Y. Ajaya Kumar, Pandit, S. Rathod, Mahadeva Swamy and Basavaraj, K. 2021. Influence of Weed Management by Allelopathy on Growth, Yield and Nutrient Status of Soil in Pigeonpea (Cajanus cajan L.). Int.J.Curr.Microbiol.App.Sci. 10(01): 2005-2018. doi: https://doi.org/10.20546/ijcmas.2021.1001.232 